\title{
Hi and Galaxy Formation in Loose Groups
}

\author{
Daniel J. Pisano ${ }^{\mathrm{A}, \mathrm{B}}$ \\ A CSIRO ATNF, Epping NSW 1710, Australia. Email: dj.pisano@csiro.au \\ B Bolton Fellow, NSF MPS Distinguished International Postdoctoral Fellow
}

Received 2004 May 3, accepted 2004 September 21

\begin{abstract}
Models of hierarchical galaxy formation predict that large numbers of low-mass, dark matter halos remain around galaxies today. These models predict an order of magnitude more halos than observed stellar satellites in the Local Group. One possible solution to this discrepancy is that the high-velocity clouds (HVCs) around the Milky Way may be associated with the excess dark matter halos and be the gaseous remnants of the galaxy formation process. If this is the case, then analogues to the HVCs should be visible in other groups. In this paper, I review the observations of Hi clouds lacking stars around other galaxies and in groups, present early results from our Hi survey of loose groups analogous to the Local Group, and discuss implications for the nature of HVCs and galaxy formation.
\end{abstract}

Keywords: Local Group — intergalactic medium — galaxies: dwarf — galaxies: formation

\section{Introduction}

Current models of hierarchical galaxy formation predict that galaxies form through the accretion of smaller lumps of gas, stars, and dark matter (e.g. Silk \& Norman 1981; Kauffman, White, \& Guiderdoni 1993; Cole et al. 1994). Recent simulations of this process assuming a lambdadominated cold dark matter (CDM) universe uniformly reveal the presence of large numbers of low-mass dark matter halos persisting around larger galaxies into the present day (e.g. Klypin et al. 1999; Moore et al. 1999). It remains unclear if these dark matter halos are filled with gas and/or stars and can be associated with dwarf galaxies and/or Hi clouds, or if they lack the mass to retain any baryons. This raises the question whether we see such gaseous remnants of galaxy formation in $\mathrm{HI}$ emission around galaxies today?

There have been many detections of Hi clouds around nearby galaxies, many of which have been hypothesised to be primordial gas associated with galaxy formation. No HI, however, has been unambiguously associated with galaxy formation. Its origin can be more readily attributed to tidal interactions, galactic fountains, or galaxy accretion.

NGC 4449 is an irregular galaxy with two counterrotating gas complexes and extended Hi distributed in large clouds, arms, and streamers (Hunter et al. 1998). While this $\mathrm{HI}$ could be explained as infalling primordial gas, the distribution and kinematics of the gas are perhaps more simply explained as resulting from a tidal interaction with a nearby dwarf galaxy (Theis \& Kohle 2001).

In NGC 6946, many clouds of HI are seen moving at velocities inconsistent with the rotation of the galaxy (Kamphuis \& Sancisi 1993). This high-velocity gas is widely associated with holes in the Hi disk, and may be explained as gas that was ejected through a galactic fountain powered by supernovae in the galaxy. An alternative explanation is that these clouds are infalling primordial material which has punched holes in the galaxy (Kamphuis \& Sancisi 1993).

There are many other cases of Hi seen outside of the main body of galaxies which have less certain origins. IC 10 (Wilcots \& Miller 1998) and NGC 925 (Pisano, Wilcots, \& Elmegreen 1998) are two examples of galaxies in groups of galaxies which have no stellar companions within $\sim 100 \mathrm{kpc}$, but both have HI clouds of $\sim 10^{7} M_{\odot}$ within a few tens of kiloparsecs. These clouds could be the remains of dwarf galaxies which have been torn apart by the larger galaxy, be tidal debris from an ancient interaction, or be primordial $\mathrm{HI}$ gas falling into these galaxies for the first time to contribute to the ongoing assembly of these galaxies.

Finally, surrounding our own galaxy are the highvelocity clouds (HVCs) - Hi clouds which lack stars and are moving at velocities inconsistent with Galactic rotation. Because of this, we cannot infer their distances or their masses (for a review see Wakker \& van Woerden 1997). HVCs most likely represent a variety of phenomena. Some HVCs are probably related to a galactic fountain (Shapiro \& Field 1976; Bregman 1980) and are located in the lower Galactic halo. Other HVCs are certainly tidal in origin: The Magellanic Stream is the most obvious of these features, formed by means of the tidal interactions between the Milky Way, Large Magellanic Cloud, and Small Magellanic Cloud (e.g. Putman et al. 1998), with other HVCs potentially related to other satellites such as the Sagittarius dwarf (Putman et al. 2004). And some HVCs, such as Complex C, may be infalling primordial gas (Wakker et al. 1999; Tripp et al. 2003; cf. Gibson et al. 2001). Finally, Blitz et al. (1999) and Braun \& Burton (1999) suggested that HVCs are the debris 
from the formation of the Local Group, and not just the Milky Way, and are associated with dark matter halos. In this scenario, HVCs are distributed throughout the Local Group with $D \sim 0.1-1 \mathrm{Mpc}$ and $M_{\mathrm{HI}} \sim 10^{5}-10^{7} M_{\odot}$.

Klypin et al. (1999) and Moore et al. (1999) raised the issue that CDM models of galaxy formation predict that the Local Group should have hundreds of small dark matter halos, while the number of known, luminous satellite galaxies is only about twenty. If HVCs are associated with dark matter halos then this discrepancy, the 'missing satellite' problem, would be resolved. Also at issue is if the Local Group is somehow unique in this regard and if other galaxies have a sufficient number of satellites to match predictions.

Unfortunately, none of the HI detections discussed above are clearly associated with galaxy formation. All of the detections may have alternative origins. To better constrain the origin of such Hi clouds, a more systematic search for them is required. I discuss here the early results of such an Hi survey of loose groups analogous to the Local Group using the Parkes Multibeam instrument and done in collaboration with David Barnes (University of Melbourne), Brad Gibson (Swinburne University), Lister Staveley-Smith (Australia Telescope National Facility), and Ken Freeman (Australian National University). This survey will determine if there are massive analogues to HVCs in other groups of galaxies associated with dark matter halos as described above. Furthermore, this survey will find gas-rich dwarf galaxies and determine if the 'missing satellite' problem is unique to the Local Group or a ubiquitous problem in all groups. Finally, these observations will serve as a benchmark for the Hi properties of galaxies in spiral-rich loose groups and how they compare to galaxies in other environments.

\section{Survey Parameters and Results}

To search for HVC analogues, to test models of galaxy formation, and to better understand the Hi properties of groups like the Local Group, we have surveyed six spiralrich, loose groups in HI 21-cm emission using the Parkes Multibeam instrument (Pisano et al. 2004a,b). A loose group is a collection of a few large galaxies and tens of smaller ones, where the large galaxies are well-separated, of order a few hundred $\mathrm{kpc}$, over an area of $\sim 1 \mathrm{Mpc}^{2}$. In contrast to compact groups, such as Stephan's Quintet and those cataloged by Hickson (1982) where interactions are a driving force, loose groups generally have few interactions occurring. We were particularly interested in loose groups containing only large spiral galaxies - groups analogous to the Local Group. If HVCs are associated with the formation of the Local Group, then they should be present in these groups as well.

The Parkes Multibeam observations were conducted in six separate observing runs between 2001 October and 2003 June. The Multibeam instrument was repeatedly scanned in right ascension and declination over an area of about $1 \mathrm{Mpc}^{2} \equiv 25 \mathrm{deg}^{2}$ with a velocity coverage of greater than $1500 \mathrm{~km} \mathrm{~s}^{-1}$ until an RMS sensitivity of
5-8 $\mathrm{mJy} \mathrm{beam}^{-1}$ per $3.3 \mathrm{~km} \mathrm{~s}^{-1}$ channel was reached. This translates to a $M_{\mathrm{HI}}$ sensitivity at the distances of these groups $(10.6-13.4 \mathrm{Mpc})$ of $(5.3-8.1) \times 10^{5} M_{\odot}$ per channel. The Parkes data had fake sources inserted before multiple double-blind searches by eye for all sources were made. All sources, not just new ones, were confirmed with follow-up Australia Telescope Compact Array (ATCA) observations. Based on our identification of fake sources, we determined that our searches were nearly $100 \%$ complete down to an integrated flux limit of ten times the RMS noise times the square root of the number of channels (Pisano et al. 2004a,b). Only three groups have had their Parkes detections confirmed with ATCA follow-up observations: LGG 93, 180, and 478. In these three groups, all twenty previously optically identified group members were detected as well as seven new HI-rich dwarf galaxies. No Hi clouds without stars identified on the Digital Sky Survey were found.

\section{Implications for High Velocity Clouds}

Because Pisano et al. (2004a) did not detect any HVC analogues in the three groups surveyed, we are unable to confirm the existence of analogues of the type proposed by Blitz et al. (1999) and Braun \& Burton (1999). We can, however, place limits on the masses and, hence, distances of the HVCs around the Milky Way if such objects are ubiquitous in the group environment. This has been done by Pisano et al. (2004b). Pisano et al. examine only the compact HVCs (CHVCs) identified by Braun \& Burton (1999) as likely being associated with dark matter halos. This is because many of the other classes of HVCs such as the Magellanic Stream and large complexes, discussed by Blitz et al. (1999) as being associated with dark matter have other, more likely, origins as discussed above. Pisano et al. assume that CHVCs are distributed in a Gaussian manner about the Milky Way, and ask for what parent population of CHVCs and what $D_{\text {HWHM }}$ for their distribution would we expect to see zero analogues around galaxies in the loose groups surveyed given the detection limits of the observations.

Figure 1 illustrates the combined constraints from the observations of LGG 93, 180, and 478 on the distances and population of CHVCs around the Milky Way. If the CHVC population in other groups has the same properties and as those around the Milky Way, then at the $95 \%$ confidence level, for 270 clouds, we see that CHVCs must be clustered within $160 \mathrm{kpc}$ of the Milky Way with an average $M_{\mathrm{HI}}$ of $\leq 4 \times 10^{5} M_{\odot}$. This is in good agreement with recent limits derived by other authors using a variety of other methods examining both Milky Way HVCs (e.g. de Heij et al. 2002) and extragalactic analogues (e.g. Zwaan 2001) and makes the original Blitz et al. (1999) and Braun \& Burton (1999) models, which place HVCs at $\sim 1 \mathrm{Mpc}$, extremely unlikely. These limits imply that CHVCs are more closely associated with individual galaxies, rather than groups of galaxies, and that there is not a large reservoir of neutral hydrogen, $\leq 1 \times 10^{8} M_{\odot}$, waiting to be accreted onto galaxies like the Milky Way. These observations do not 


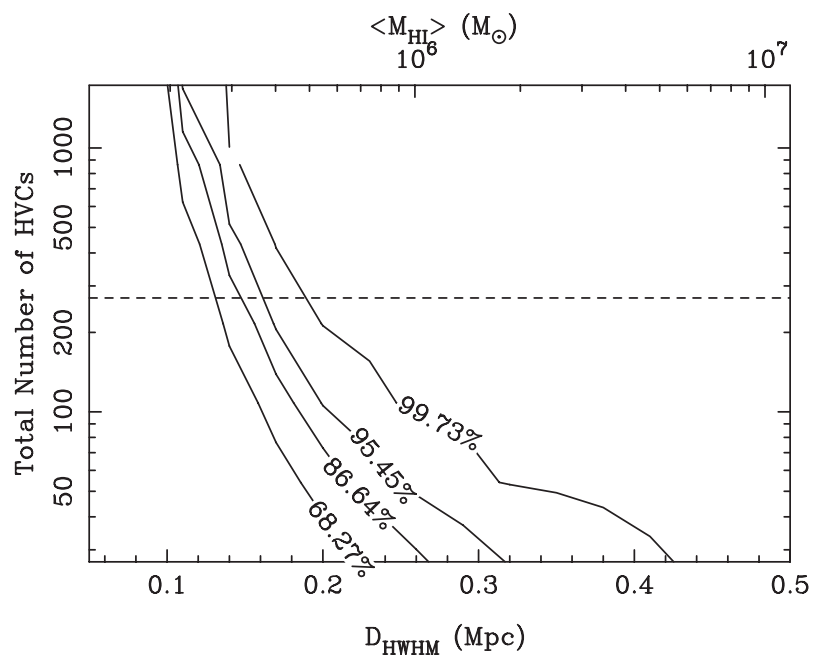

Figure 1 A plot of the combined probability of zero detections in LGG 93, 180, and 478 as a function of the number of CHVCs per group and $D_{\text {HwHM }}$ (equivalent to the average $M_{\mathrm{HI}}$ of a CHVC) for the distribution of Milky Way CHVCs. The dashed line marks the number of CHVCs identified around the Milky Way.

rule out the presence of a large reservoir of ionised gas, however.

\section{Comparison with Models of Galaxy Formation}

The observations of loose groups by Pisano et al. (2004a) do not find large numbers of HI-rich galaxies that would correspond to the low mass dark matter halos seen in the simulations by Klypin et al. (1999) or Moore et al. (1999). As such we can infer that the Local Group is not unique in its lack of low mass, luminous satellites as compared to the predictions of CDM simulations. Pisano \& Wilcots (2003) previously found this to be the case for gas-rich companions to isolated galaxies. These results are illustrated in Figure 2.

The cumulative velocity distribution functions for the Local Group, loose groups, and isolated galaxies have roughly consistent slopes which are inconsistent with the CDM models. From this it is clear that our measurements of luminous halos do not match CDM predictions and either an alternative form of dark matter (such as warm dark matter, Colín et al. 1999) or a mechanism suppressing the collapse of baryons into dark halos (e.g. Tully et al. 2002) is needed to reconcile the observations with models. These results will be discussed in more detail in future papers (Pisano et al., in preparation).

\section{Acknowledgements}

I would like to thank the organisers of the 'Tullyfest' for inviting me to present this work at such an excellent meeting. I would also like to thank the staff at Parkes and the ATCA for their wonderful support of these observations. I would like to acknowledge generous support from both an ATNF Bolton Fellowship and NSF MPS Distinguished International Research Fellowship grant AST0104439.

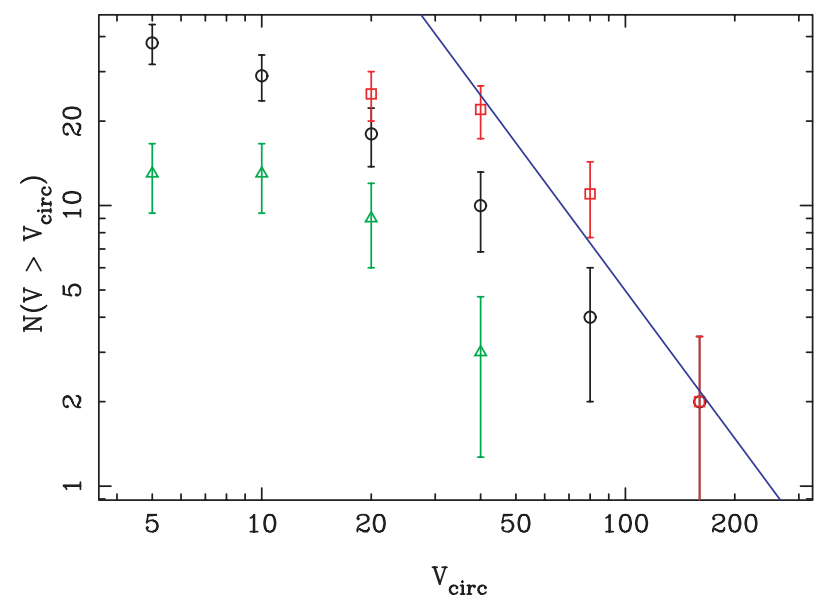

Figure 2 A plot of the cumulative velocity distribution function for the Local Group (black circles), our three loose groups (red squares), and companions to isolated galaxies (green triangles). Error bars indicate $1 \sigma$ Gaussian errors. The solid blue line is the prediction for CDM models of galaxy formation from Klypin et al. (1999) with arbitrary normalisation.

\section{References}

Blitz, L., Spergel, D., Teuben, P. J., Hartmann, D., \& Burton, W. B. 1999, ApJ, 514, 818

Braun, R., \& Burton, W. B. 1999, A\&A, 341, 437

Bregman, J. N. 1980, ApJ, 236, 577

Cole, S., Aragón-Salamanca, A., Frenk, C. S., Navarro, J. F., \& Zepf, S. E. 1994, MNRAS, 271, 781

Colín, P., Avila-Reese, V., \& Valenzuela, O. 2000, ApJ, 542, 622

de Heij, V., Braun, R., \& Burton, W. B. 2002, A\&A, 392, 417

Gibson, B. K., Giroux, M. L., Penton, S. V., Stocke, J. T., Shull, J. M., \& Tumlinson, J. 2001, AJ, 122, 3280

Hickson, P. 1982, ApJ, 255, 382

Hunter, D. A., Wilcots, E. M., van Woerden, H., Gallagher, J. S., \& Kohle, S. 1998, ApJ, 495, L47

Kamphuis, J., \& Sancisi, R. 1993, A\&A, 273, L31

Kauffman, G., White, S. D. M., \& Guiderdoni, B. 1993, MNRAS, 264, 201

Klypin, A., Kravtsov, A. V., Valenzuela, O., \& Prada, F. 1999, ApJ, 522,82

Moore, B., Ghigna, S., Governato, F., Lake, G., Quinn, T., Stadel, J., \& Tozzi, P. 1999, ApJ, 524, L19

Pisano, D. J., \& Wilcots, E. M. 2003, ApJ, 584, 228

Pisano, D. J., Wilcots, E. M., \& Elmegreen, B. G. 1998, AJ, 115, 975

Pisano, D. J., Barnes, D. G., Gibson, B. K., Staveley-Smith, L., \& Freeman, K. C. 2004a, IAU Symp. 217, eds. P.-A. Duc, J. Braine, \& E. Brinks, 20

Pisano, D. J., Barnes, D. G., Gibson, B. K., Staveley-Smith, L., Freeman, K. C., \& Kilborn, V. A. 2004b, ApJ, 610, L17

Putman, M. E., et al. 1998, Natur, 394, 752

Putman, M. E., Thom, C., Gibson, B. K., \& Staveley-Smith, L. 2004, ApJ, 603, L77

Shapiro, P. R., \& Field, G. B. 1976, ApJ, 205, 762

Silk, J., \& Norman, C. 1981, ApJ, 247, 59

Theis, C., \& Kohle, S. 2001, A\&A, 370, 365

Tripp, T. M., et al. 2003, AJ, 125, 3122

Tully, R. B., Somerville, R. S., Trentham, N., \& Verheijen, M. A. W. 2002, ApJ, 569, 573

Wakker, B. P., \& van Woerden, H. 1997, ARA\&A, 35, 217

Wakker, B. P., et al. 1999, Natur, 400, 388

Wilcots, E. M., \& Miller, B. W. 1998, AJ, 116, 2363

Zwaan, M. A. 2001, MNRAS, 325, 1142 